\title{
An Unexpected Operative Finding of Intracerebral Pus in a Case of Multifocal Cerebral Metastases from Small Cell Lung Cancer
}

\author{
Ibiwari Pepple* and Debasish Hajra \\ Department of Neurosurgery, University Hospital Coventry and Warwickshire, West Midlands, United \\ Kingdom
}

*Corresponding author: Ibiwari Pepple, Department of Neurosurgery, University Hospital Coventry and Warwickshire, Coventry, West Midlands, United Kingdom

\begin{abstract}
Background: Small cell lung cancer (SCLC) accounts for $10-20 \%$ of all lung cancers and is characterised by early and rapid spread. Ten percent of patients already have cerebral metastases at time of presentation.

Case description: We report a case of a 37-yr-old female whose pre-operative CT and MRI brain revealed multicentric left frontal and temporal lesions which mimicked intracerebral abscess intraoperatively and confirmed on gram stain as moderate growth of white cells. The patient was commenced on prolonged antimicrobial therapy postoperatively, however, histology reported SCLC metastases with lung as primary and patient was eventually commenced on chemotherapy.

Conclusion: The prevalence of intraoperative histopathological frozen section to better support diagnosis and good understanding of SCLC cerebral metastases from accumulation of cases together with modern diagnostic tools will hopefully lead to prompt and adequate therapeutic options for patients.
\end{abstract}

\section{Keywords}

Antimicrobial therapy, Cerebral abscess, Multifocal cerebral metastasis, Small cell lung cancer

\section{Introduction}

Lung cancer is undoubtedly the most common cause of cancer related deaths in the United Kingdom and worldwide for both men and women, accounting for one-fifth of all cancer deaths.

Forty percent of patients with lung cancer have brain metastases (highest amongst all malignant tu- mours), of which $48 \%$ have single metastasis and $52 \%$ multiple of multifocal.

SCLC account for $10-20 \%$ of all lung cancers. Histologically, it is the third most common subtype but has the highest malignant potential. And it has been reported that at time of initial admission, approximately $10 \%$ of all SCLC patients have cerebral metastases [1]. Several papers have reported as high as $80-90 \%$ of supratentorial metastases from SCLC of which $60-70 \%$ have multiple characteristics [2].

SCLC spread early via intrathoracic and mediastinal lymph nodes to brain, liver, bone etc. Clinically, patients will complain of headache, vomiting, sensorimotor deficits, seizures, behavioural problems and aphasia. However, these symptoms can be subtle resulting in late hospital presentation.

\section{Case Report}

A 37-year-old female who spoke little or no English presented with 2 wks history of headache, blurry vision, slurred speech, dizziness, confusion but no sensorimotor deficits. She had no significant medical history prior to her hospital admission and socially she confirmed that she is a regular smoker.

A non-enhanced computed tomography (NECT) of the head (Figure 1) showed large hypodense mass $(5 \times$ $6 \mathrm{~cm}$ ) in the left frontal lobe with hyperdense margin crossing midline and compressing the frontal horn of left lateral ventricle. Our differential diagnosis centred around glial based tumour with very little possibility of

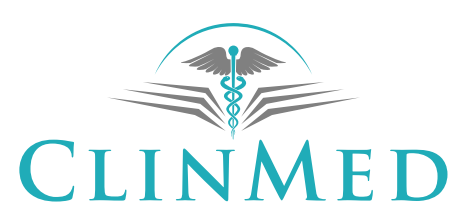

INTERNATIONAL LIBRARY

Citation: Pepple I, Hajra D (2019) An Unexpected Operative Finding of Intracerebral Pus in a Case of Multifocal Cerebral Metastases from Small Cell Lung Cancer. Neurosurg Cases Rev 2:024. doi. org/10.23937/2643-4474/1710024

Accepted: October 03, 2019; Published: October 05, 2019

Copyright: (C) 2019 Pepple I, et al. This is an open-access article distributed under the terms of the Creative Commons Attribution License, which permits unrestricted use, distribution, and reproduction in any medium, provided the original author and source are credited. 


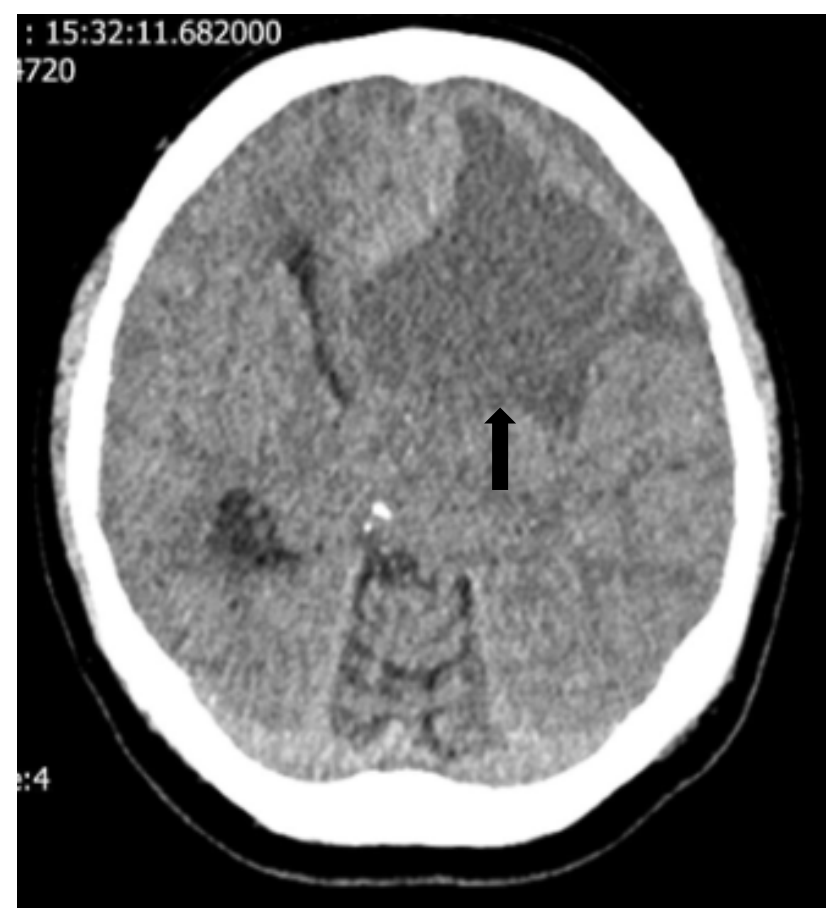

Figure 1: Shows hypodense lesion (black arrow) compressing the left frontal horn.

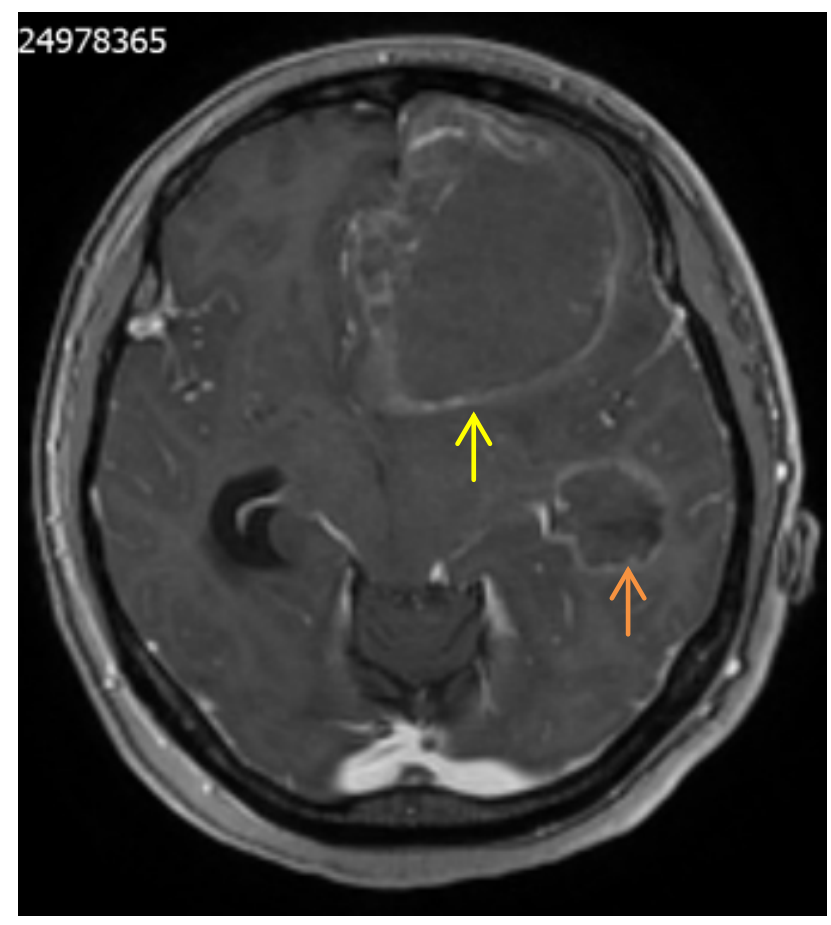

Figure 2: Shows multicentric lesions in the left frontal (yellow arrow) and temporal (orange arrow) lobes with rim enhancement.

an abscess based on imaging features.

She had routine bloods which showed a white cell of $94,300 / \mathrm{mm}^{3}, \mathrm{Hb}$ of $150 \mathrm{~g} / \mathrm{L}$, normal BUN and electrolyte level. Patient was also commenced on high dose dexamethasone and levetiracetam for intracerebral edema and seizure prophylaxis.

As per our unit protocol, she underwent contrast enhanced magnetic resonance imaging (CE-MRI) of brain

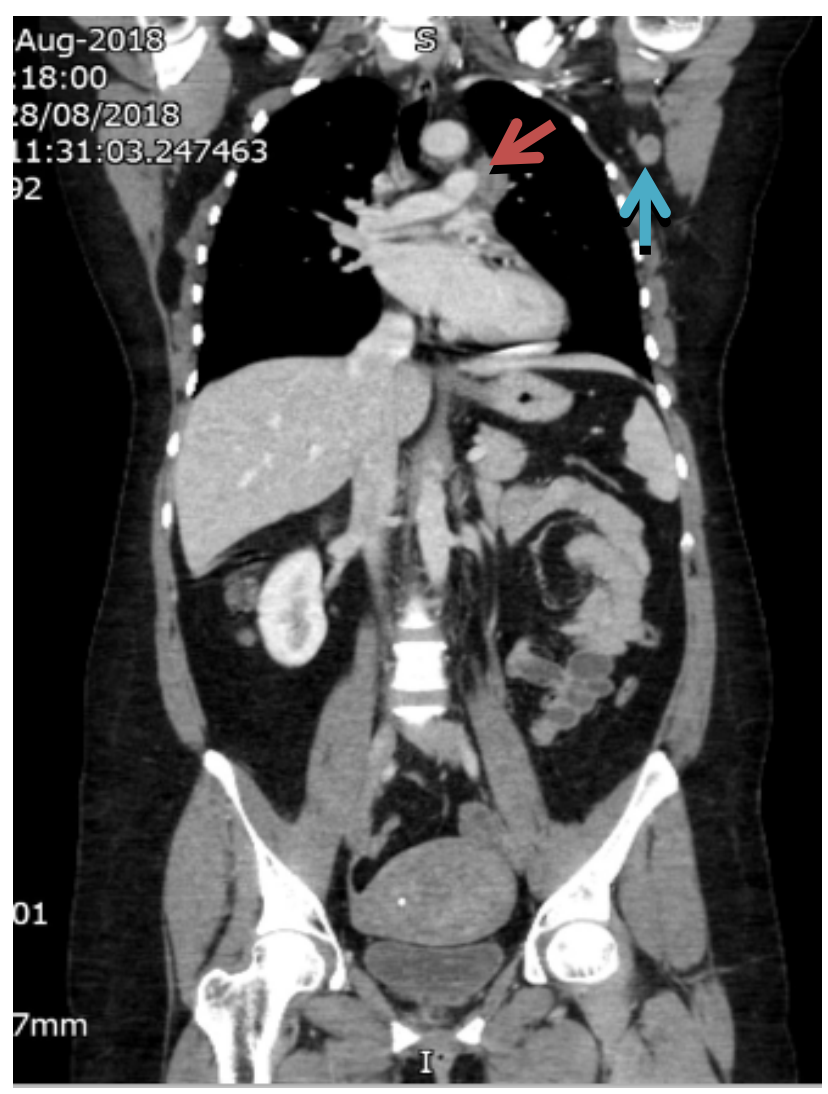

Figure 3: Shows left hilar lymph node abutting left pulmonary artery (brown arrow) and in the left axilla (blue arrow).

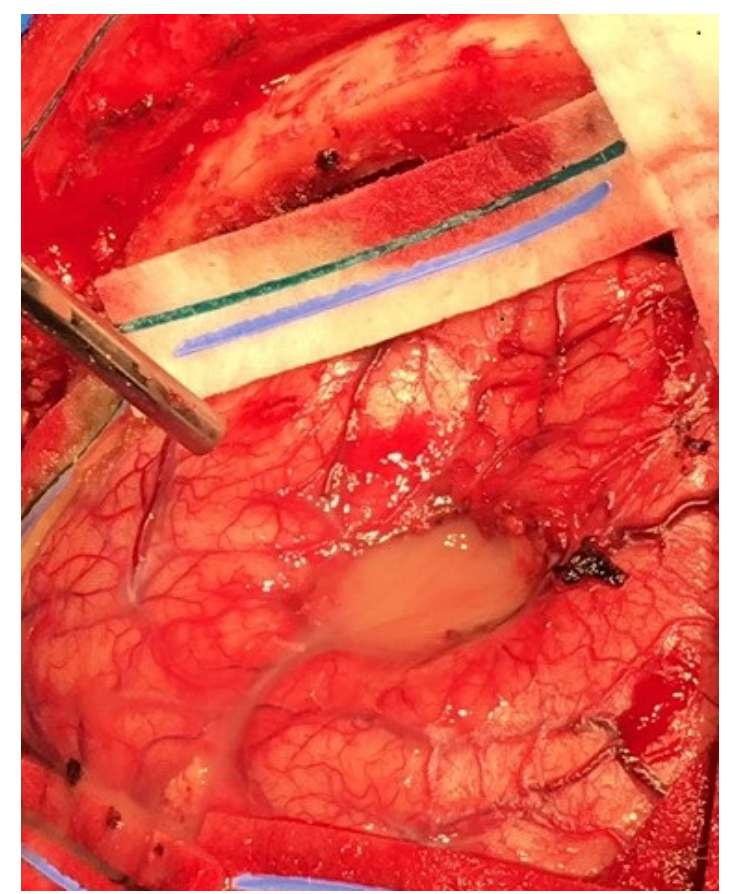

Figure 4: Intraoperative appearance of the left frontal lesion showing frank purulent fluid in the cavity.

inclusive of stereotactic planning sequence (Figure 2), CT chest, abdomen and pelvis (Figure 3).

MRI was reported as multicentric lesion likely primary glioma and metastatic deposits as possible differentials, but unlikely to be an abscess due to lack of diffusion restriction in the centre of the lesions. CT 
chest, abdomen and pelvis (CAP) found incidental left hilar and axillary adenopathy for which guided biopsy was advised but was not followed up on at this point.

The case was discussed in the neuro-oncology multidisciplinary team (MDT) meeting and it was recommended that she undergo tumour debulking.

Intraoperatively after stealth registration and craniotomy, corticotomy done directly above the lesion revealed yellowish fluid suggestive of frank pus gushing out of the suspected tumour cavity (Figure 4). Samples were immediately sent to the laboratory for urgent microscopy, culture and sensitivity. A portion of the presumed abscess cavity wall was also sent for histology. It is important to mention that the temporal lobe lesion was not interfered with during surgery.

She was commenced on intravenous meropenem intraoperatively as empirical treatment based on intraoperative findings During her recovery, further investigations in the form of echocardiogram, OPG x-ray to look for potential source was carried out. These investigations did not reveal the source of the abscess and though microscopy of the purulent fluid showed moderate growth of white blood cells, no organisms were isolated on culture.

Her post-operative contrast enhanced CT showed reduction in size of the lesion (Figure 5). However, the recovery period was complicated by few episodes of seizures and CT venogram confirmed left transverse sinus thrombosis, both of which were adequately treated.

Based on the intraoperative findings, we had so-

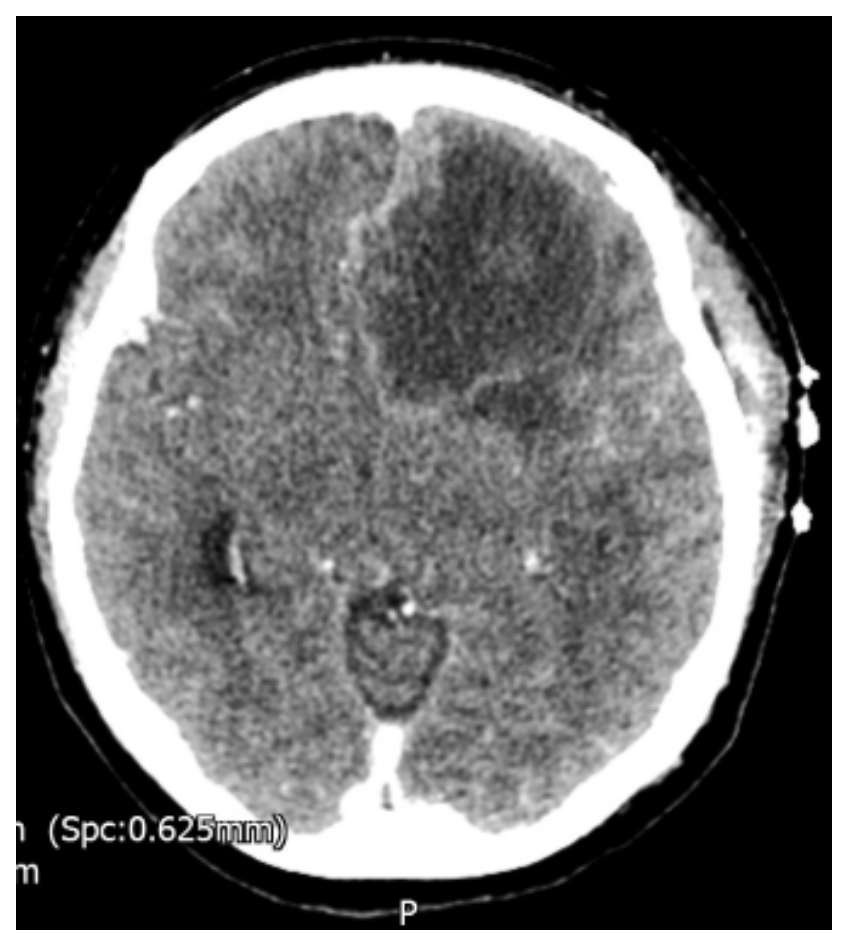

Figure 5: Shows post-operative cavity with surrounding edema. mewhat agreed that this is likely an intracerebral cerebral abscess of unknown source for which antibiotics was started following standard guidelines.

However, the histology was positive for metastatic small cell carcinoma and Immunohistochemistry favoured a primary in the lung. In the view of the histology report, patient was referred to the lung MDT for further discussion and management.

\section{Discussion}

MRI is the modality of choice for differentiating brain metastases from brain abscess and glioblastoma. On diffusion weighted imaging (DWI) the central area of an abscess is hyper intense due to high viscosity which differentiates it from brain tumours [3]. A recent report by fink, et al. [4] indicated that brain metastases tend to show high apparent diffusion coefficient (ADC) values while DWI will distinguish between metastases and abscess with high diffusion restriction. This was in contrast to an earlier study by Duygulu, et al. [5] which reported that increased diffusion restriction for both metastatic SCLC and NSCLC. Even with large studies on lung cancer brain metastases, there are no established trends in MRI manifestations as compared to metastases from other regions (melanoma, kidney and colon) that MRI pathognomonic findings have been reported.

In this case and due to the ambiguity as highlighted in the studies above, the intraoperative findings where very much in keeping with intracerebral pus collection (Figure 4) cascading into sample collection for culture and sensitivity, with post-operative antimicrobial therapy.

Retrospectively, maybe the use of additional MR sequences like susceptibility weighted imaging (SWI), proton magnetic resonance spectroscopy $\left({ }^{1} \mathrm{H}-\mathrm{MRS}\right)$, whole body diffusion weighted MRI (DW-MRI) or an additional modality like positron emission tomography (PET) would have changed the approach of giving postoperative antibiotics in this case.

Also, the CT CAP which revealed hilar and mediastinal adenopathy could have been biopsied to attain a diagnosis and potentially prevent antibiotic use. However, we need to stress that that the patient was already symptomatic from the mass effect of the lesion which is the main reason operative intervention was pursued as a matter of urgency and one which yielded good post-operative improvement in neurological deficit.

\section{Conclusion}

This case report has highlighted an unexpected intra-operative finding of intracerebral pus in multifocal SCLC cerebral metastasis which convinced the surgical team to pursue the use of antimicrobial therapy and to 
further investigate the source of the abscess only for histopathological examination to confirm the diagnosis as metastatic SCLC.

We would like to suggest a robust use of available investigatory armamentarium to clearly differentiate cerebral abscess from metastasis when dealing with ambiguous cases. Secondly, emphasis has to be placed on the importance of frozen section diagnosis which our team believe should be made widely available in other to assist with intraoperative diagnosis and prevent exposing patients to extra investigations and treatment as in this case.

\section{References}

1. Auchter RM, Lamond JP, Alexander E, Buatti JM, Chappell $R$, et al. (1996) A multiinstitutional outcome and prognostic factor analysis of radiosurgery for resectable single brain metastasis. Int J Radiat Oncol Biol Phys 35: 27-35.

2. Dolgushin Mikhail, Kornienko Valery, Pronin Igor (2018) Brain Metastases: Advanced neuroimaging.

3. Saito T, Ujiie H, Kadoyama S, Higa T, Shiono S, et al. (2015) Brain metastasis from a lung mucoepidermoid carcinoma mimicking a brain abscess. Surg Neurol Int.

4. Fink KR, Fink JR (2013) Imaging of $b$ rain metastases. Surg Neurol Int 4: S209-S219.

5. Duygulu G, Ovali GY, Calli C, Kitis O, Yunten N, et al. (2010) Intracerebral metastasis showing restricted diffusion: Correlation with histopathologic findings. Eur J Radiol 74: 117-120. 